\title{
Improving the classification of brain tumors in mice with perturbation enhanced (PE)-MRSI
}

\author{
Rui V Simões ${ }^{1,2^{*}}$, Sandra Ortega-Martorell ${ }^{1,2,3}$, Teresa Delgado-Goñi ${ }^{1,2}$, Yann le Fur ${ }^{4}$, Marti Pumarola ${ }^{5}$, \\ Ana P Candiota ${ }^{1,2}$, Patrick J Cozzone ${ }^{4}$, Margarida Juliá-Sapè ${ }^{1,2,3}$, Carles Arús , $^{1,2,3}$ \\ From 16th International Charles Heidelberger Symposium on Cancer Research \\ Coimbra, Portugal. 26-28 September 2010
}

Classifiers based on pattern recognition analysis of MRS(I) data are becoming important tools for the noninvasive diagnosis of human brain tumors [1,2]. Here we investigate the potential interest of perturbation-enhanced MRSI (PE-MRSI), in this case acute hyperglycemia, during moderate brain hypothermia $[3,4]$, for improving the discrimination between mouse brain MRS patterns of glioblastoma multiforme (GBM), low grade oligodendroglioma (ODG2), and non-tumor brain parenchyma (NT). Six GBM-bearing mice and two ODG2-bearing mice were scanned at 7 Tesla by PRESS-MRSI with 12 and $136 \mathrm{~ms}$ echo-time, during euglycemia (Eug) and also during induced acute hyperglycemia (Hyp), generating altogether four datasets per animal (echo time + glycemic condition): $12 \mathrm{Eug}, 136 \mathrm{Eug}, 12 \mathrm{Hyp}$, and $136 \mathrm{Hyp}$. For classifier development, using in-house built software SpectraClassifier 2.0 [5,6], all spectral vectors (spv) selected were unit length normalized (UL2) and used either as training set (76 GBM spv, four mice; 70 ODG2 spv, 2 mice; 54 NT spv, 6 mice) or as independent test set (2 mice, 61 GBM spv and 17 NT spv). All Fisher's LDA classifiers obtained had very good descriptive performance when extracting at least 10 features from the training sets as evaluated by Bootstrapping: correctly classified cases $\geq 99 \%$. Evaluation of predictive performance with the independent test set clearly revealed that 12Hyp MRSI-based classifiers with at least 5 features provided the best robustness: balanced error rate (BER) for spv prediction $<0.9 \%$. This highlights the potential interest of perturbation-enhanced MRSI protocols for improving the non-invasive characterization of brain tumors at a preclinical level.

\section{Author details}

'Bioquímica i Biologia Molecular, Facultat de Biociències, Universitat Autònoma de Barcelona, Barcelona, Spain. ${ }^{2}$ Centro de Investigación Biomédica en Red - Bioingeniería, Biomateriales y Nanomedicina (CIBERBBN), Spain. Institut de Biotecnologia i de Biomedicina, Universitat Autònoma de Barcelona, Barcelona, Spain. ${ }^{4}$ Centre de Résonance Magnétique Biologique et Médicale (CRMBM) UMR CNRS 6612, Marseille, France. ${ }^{5}$ Banc de Teixits Animals de Catalunya, Departament de Medicina i Cirurgia Animals, Universitat Autònoma de Barcelona, Barcelona, Spain.

Published: 24 September 2010

\section{References}

1. De Edelenyi FS, Rubin C, Estève F, Grand S, Décorps M, Lefournier V, Le Bas J-F, Rémy C: A new approach for analyzing proton magnetic resonance spectroscopic images of brain tumors: nosologic images. Nat Med 2000, 6:1287-1289

2. Tate AR, Underwood J, Acosta DM, Julià-Sapé M, Majós C, Moreno-Torres A, Howe FA, van der Graaf M, Lefournier V, Murphy MM, Loosemore A, Ladroue C, Wesseling P, Luc Bosson J, Cabañas ME, Simonetti AW, Gajewicz W, Calvar J, Capdevila A, Wilkins PR, Bell BA, Rémy C, Heerschap A, Watson D, Griffiths JR, Arús C: Development of a decision support system for diagnosis and grading of brain tumours using in vivo magnetic resonance single voxel spectra. NMR Biomed 2006, 19:411-434.

3. Simões RV, García-Martín ML, Cerdán S, Arús C: Perturbation of mouse glioma MRS pattern by induced acute hyperglycemia. NMR Biomed 2008, 21:251-264.

4. Simões RV, Delgado-Goñi T, Lope-Piedrafita S, Arús C: $1 \mathrm{H}-\mathrm{MRSI}$ pattern perturbation in a mouse glioma: the effects of acute hyperglycemia and moderate hypothermia. NMR Biomed 2010, 23:23-33.

5. Ortega-Martorell $S$, Olier I, Juliá-Sapè M, Arús C: SpectraClassifier 1.0: a user friendly, automated MRS-based classifier-development system. BMC Bioinformatics 2010, 11:106.

6. SpectraClassifier (SC). [http://gabrmn.uab.es/SC].

doi:10.1038/81401

Cite this article as: Simões et al:: Improving the classification of brain tumors in mice with perturbation enhanced (PE)-MRSI. BMC Proceedings 2010 4(Suppl 2):P65.

\footnotetext{
* Correspondence: simoesr@mskcc.org

'Bioquímica i Biologia Molecular, Facultat de Biociències, Universitat

Autònoma de Barcelona, Barcelona, Spain

Full list of author information is available at the end of the article
} 